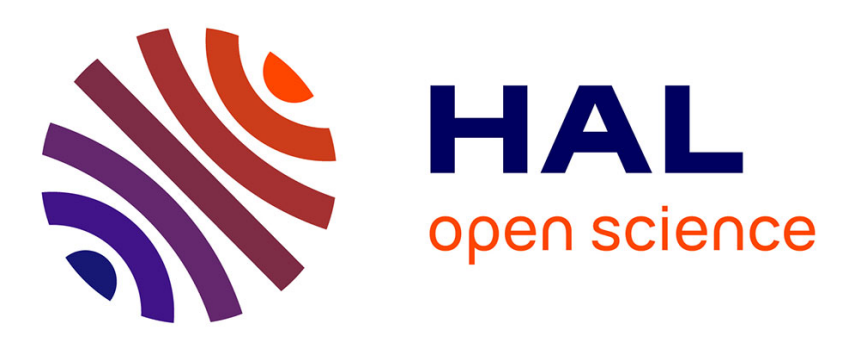

\title{
Une distance sociale intime. Hétérogamie parentale et choix de l'agriculture par les fils et filles d'agriculteur
}

Christophe Giraud

\section{To cite this version:}

Christophe Giraud. Une distance sociale intime. Hétérogamie parentale et choix de l'agriculture par les fils et filles d'agriculteur. Fabrice Boudjaaba. Le travail et la famille en milieu rural(XVIe-XXe siècle) , PUR, pp.185-206, 2013. hal-01460736

\section{HAL Id: hal-01460736 \\ https://hal-univ-paris.archives-ouvertes.fr/hal-01460736}

Submitted on 19 Jun 2017

HAL is a multi-disciplinary open access archive for the deposit and dissemination of scientific research documents, whether they are published or not. The documents may come from teaching and research institutions in France or abroad, or from public or private research centers.
L'archive ouverte pluridisciplinaire HAL, est destinée au dépôt et à la diffusion de documents scientifiques de niveau recherche, publiés ou non, émanant des établissements d'enseignement et de recherche français ou étrangers, des laboratoires publics ou privés. 


\title{
Une distance sociale intime Hétérogamie parentale et choix de l'agriculture par les fils et filles d'agriculteur
}

\author{
Christophe Giraud
}

\section{Introduction}

Une grande transformation bouleverse l'agriculture française depuis le début des années 1970 : les femmes ont de plus en plus une activité professionnelle salariée, indépendante de celle de leur conjoint agriculteur. Quelle que soit l'interprétation donnée à ce phénomène, la montée de "l'hétérogamie professionnelle » dans les couples en agriculture est un phénomène massif et croissant. En 2000, $43 \%$ des conjointes d'agriculteurs se déclaraient agricultrices en activité. Elles étaient $54 \%$ dix ans auparavant. En 2003, $75 \%$ des conjointes de moins de 25 ans n'avaient aucune activité sur l'exploitation ${ }^{1}$.

Ce phénomène marque une double rupture: la première renvoie à l'image que les sciences sociales se sont faites de l'univers agricole, socialement caractérisé par une forme de clôture tant géographique que sociale. Les paysans étaient d'abord définis comme membres d'une communauté locale et soumis aux normes et à la culture en partie autonome du groupe local. Les unions conjugales se faisaient d'abord " en interne ». Elles avaient pour fonction d'assurer la reproduction non seulement de la position sociale des lignées dans le groupe local, mais aussi celle du groupe local tout entier. La seconde rupture renverse la politique des structures menée depuis les années 1960 qui privilégiait un modèle d'agriculture familiale dit à deux UTH (unité de travail humain) ${ }^{2}$ où le couple formait l'équipe de

1. L'hétérogamie en agriculture concerne plutôt les exploitations spécialisées, tout particulièrement les céréaliers, alors que la situation d'homogamie en agriculture est plus souvent le fait des exploitations peu spécialisées, en polyculture-élevage (Allaire G., Blanc M., Pluriactivité et trajectoires professionnelles des agriculteurs, Document de travail, $\mathrm{n}^{\circ}$ WP91-04, 1991, 42 p. et Giraud C., Rémy J., " Les choix des conjoints en agriculture ", Revue d'études en agriculture et environnement, $\mathrm{n}^{\circ} 3,2008$, p. 21-46).

2. Remy J., "Introduction ", in Hervieu B., Mayer N., Muller P., Purseigle F., Remy J. (dir.), Les mondes agricoles en politique, Paris, Les Presses de Sciences Po, 2010, p.41-50. 
travail par excellence sur une exploitation destinée à faire vivre une famille conjugale ${ }^{3}$.

Désormais les couples en agriculture peuvent vivre dans une situation intime de mixité sociale: aux côtés d'hommes agriculteurs, des femmes peuvent disposer d'un travail salarié qu'elles ont choisi et auquel elles s'identifient fortement. Les rythmes des activités agricoles et salariées peuvent être très différents (les week-ends, les vacances scolaires peuvent devenir un problème), et les capitaux culturels dont disposent chacun peuvent être très dissemblables. La situation d'hétérogamie croissante interroge la capacité de l'univers agricole à se reproduire d'une génération à l'autre ${ }^{4}$, c'est-à-dire à former des individus dotés des dispositions adaptées à cet univers professionnel et social et susceptibles de s'y inscrire durablement.

Nous formulons ici l'hypothèse selon laquelle la socialisation d'un individu dans une famille dont les deux parents travaillent sur l'exploitation (éventuellement en tant qu'agriculteur ou salarié agricole) contribue à entretenir un sentiment d'évidence sur le monde social et professionnel, sur les rythmes de travail, les valeurs dans la vie et les ambitions sociales et professionnelles, ce que la socialisation dans une famille où deux mondes professionnels cohabitent ne permet pas d'assurer avec autant de force. Pour autant, l'effet de l'hétérogamie n'est en rien uniforme mais dépend de la «valeur sociale » de la profession non-agricole d'un des deux parents en comparaison de celle de la profession agricole. L'importance économique de l'exploitation agricole parentale doit donc être prise en compte dans cette évaluation de la position sociale relative des deux conjoints 5 .

Si la clôture sociale parentale favorise le goût pour l'agriculture par rapport à une certaine mixité, il faut aussi qu'elle apporte également les outils indispensables pour devenir agriculteur ou agricultrice de manière effective. Or, l'entrée dans le monde agricole s'est complexifiée durant la même période: il n'est plus autant un destin qui obligerait l'aîné, ou un autre membre masculin, à reprendre l'exploitation familiale. Il est aujourd'hui fortement "médié " par la profession qui, depuis 1974, conditionne ses aides à l'installation à l'obtention d'un certain niveau de diplôme ${ }^{6}$. Si la famille reste centrale dans la transmission des capitaux indis-

3. D'évidence, la " déconjugalisation " des exploitations familiales progresse fortement, et elle est particulièrement élevée dans le secteur des grandes cultures (Dufour A., Giraud C., " Le travail dans les exploitations d'élevage bovin laitier est-il toujours conjugal? ", in Hostiou N., Dedieu B., Baumont R. (eds.), numéro "Travail en élevage ", INRA-Productions animales, 25, 2, juin, 2012, p. 169-180).

4. Sur les ajustements qui se font au quotidien dans le couple pour accorder des mondes sociaux en partie hétérogènes, voir la thèse d'Isabelle van de WaLLE, Terre des hommes, salaire des femmes, Paris, EHESS, sous la direction de Bertrand Hervieu, 1993.

5. La petite agriculture peu spécialisée a ainsi montré des signes très forts de découragement, de volonté de ne pas se reproduire, privilégiant ainsi pour leurs enfants des trajectoires professionnelles en direction du monde salarié (Champagne P., L'héritage refusé, Paris, Seuil, 2002).

6. MARESCa S., "La réussite d'une politique. Interrogations sur les effets sociaux des incitations publiques ", Cahier d'économie et de sociologie rurale, nº 6, 1988, p. 5-40. 
pensables à une reprise, l'école devient de plus en plus une institution incontournable par laquelle les enfants d'agriculteurs doivent passer avant de s'installer ${ }^{7}$. C'est une institution qui permet notamment aux jeunes femmes d'acquérir une formation agricole quand la socialisation au métier au sein de la famille leur était généralement refusée lorsqu'un frère montrait de l'intérêt pour l'agriculture ${ }^{8}$. Si des jeunes femmes s'installent aujourd'hui plus fréquemment en agriculture ${ }^{9}$, elles sont encore peu nombreuses en raison de la priorité donnée aux fils dans les processus de désignation de successeur et nous verrons que l'entrée dans le métier d'agricultrice des filles d'agriculteurs passe encore beaucoup par le mariage.

La première partie sera consacrée à la façon dont la situation d'hétérogamie des parents influence le niveau scolaire des enfants, l'engagement matrimonial des filles et le choix de devenir agriculteur. Une seconde partie essaiera d'analyser l'impact de la position sociale du parent non agriculteur sur le capital scolaire des enfants, l'engagement matrimonial féminin et le choix de l'agriculture.

\section{Les données utilisées et la mesure de l'homogamie/hétérogamie}

Nous essaierons de vérifier nos hypothèses en nous appuyant principalement sur les données de l'enquête FQP réalisée par l'INSEE en 2003 auprès d'un échantillon de presque 40000 personnes de plus de 15 ans dont 502 hommes agriculteurs et 237 femmes agricultrices et 2348 fils et 2357 filles d'un père ou d'une mère agriculteur (soit une population totale de 4705 fils ou filles ayant au moins un parent agriculteur). Nous avons également utilisé l'Enquête sur l'Histoire Familiale (EHF) réalisée en 1999 par l'INSEE ${ }^{10}$ dans la seconde partie car elle nous permettait d'analyser, avec des effectifs plus confortables ${ }^{11}$, les trajectoires scolaires des enfants d'agriculteurs en fonction de la profession de la mère.

Les conditions optimales d'une analyse de la mobilité intergénérationnelle consistent à effectuer une comparaison de la position des parents et de celle des enfants à peu près au même âge. La profession des parents ayant été mesurée au moment de la fin des études de la personne interrogée, c’est-

7. Giraud C., Rémy J., à paraître.

8. Bessière C., De génération en génération. Arrangements de famille dans les entreprises viticoles de Cognac, Paris, Raisons d'agir, 2010.

9. «En 2003, la part des femmes parmi les exploitants professionnels de moins de 40 ans est de $17 \%$. Elles représentent un jeune co-exploitant en société sur trois, mais seulement un jeune chef d'exploitation individuelle sur dix " (Bessière C., op. cit., p. 97).

10. Étude de l'histoire familiale (EHF) - 1999 - (1999) [fichier électronique], et Formation et Qualification professionnelle (FQP) - 2003 - (2003) [fichier électronique], INSEE [producteur], Centre Maurice Halbwachs (CMH) [diffuseur].

11. 4005 fils et 5893 filles d'un père ou d'une mère se déclarant agriculteur, âgés de 40 à 49 ans au moment de l'enquête. 
à-dire entre 16 et 30 ans, l'âge des pères va donc se situer dans une fourchette comprise entre 36 et 60 ans. On a choisi de centrer le plus possible nos analyses sur la cohorte des individus de 40 à 49 ans (soit environ 4303 hommes et 4794 femmes dont 602 fils et 560 filles ayant un père ou une mère agriculteur) car il s'agit d'une classe d'âge où la position sociale des individus interrogés est relativement stable (plus de détours professionnels) et dont (en raison de l'étroitesse de la classe) les conditions de socialisation familiale, d'études et d'opportunités sur le marché du travail sont assez homogènes. Cependant les contraintes statistiques ont souvent imposé de prendre en compte l'ensemble de la population des fils et des filles afin d'obtenir des résultats significatifs.

L'influence de l'homogamie ou de l'hétérogamie sur le devenir scolaire et professionnel des enfants d'agriculteur doit essayer de prendre en compte les opportunités différenciées de "succéder " selon l'importance économique des exploitations agricoles. Quatre situations familiales peuvent ainsi être distinguées ${ }^{12}$ :

- les enfants de parents hétérogames travaillant sur une petite exploitation: $15,1 \%$ de la sous-population des fils et des filles en 2003;

- les enfants de parents homogames travaillant sur une petite exploitation : $65,8 \%$;

- les enfants de parents hétérogames travaillant sur grande ou moyenne exploitation : 3,9\%;

- les enfants de parents homogames travaillant sur grande et moyenne exploitation : $15,3 \%$.

Pour la génération des enfants âgés de 40 à 49 ans (individus nés entre 1954 et 1963), avoir deux parents agriculteurs est toujours beaucoup plus fréquent que d'en avoir un seul $(82,8 \%$ contre $17,2 \%)$ car le marché matrimonial des parents qui se sont mariés avant 1963 restait encore assez fermé et que les contraintes normatives de la profession agricole pesaient fortement sur les réorientations professionnelles des femmes au moment de leur mariage. Pour les générations plus jeunes (hommes nés entre 1964 et 1973, âgés de moins de 30 à 39 ans en 2003), l'importance de l'homogamie parentale se réduit $(75,2$ contre $24,8 \%)$. Et pour les fils d'agriculteurs nés après 1974 (âgés de moins de 30 ans en 2003), l'hétérogamie fait presque jeu égal chez les enfants d'agriculteur ( 56,8 contre $45,2 \%$ ), ce qui montre les transformations très importantes de la position des agriculteurs sur le marché matrimonial depuis les années $1960^{13}$.

Le fait d'avoir deux parents agriculteurs est significativement plus important dans les grandes et moyennes exploitations que dans les petites (20,1\% des enfants de tous âges, issus d'exploitations grandes ou moyennes contre $18,7 \%$ des enfants issus de petites exploitations). L'importance économique des exploitations, élément très important de la valeur sociale des agriculteurs sur le marché matrimonial, favorise l'hétérogamie.

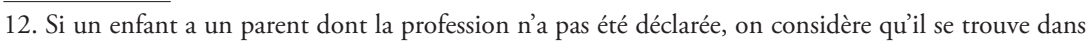
une famille homogame.

13. Giraud C., RÉmy J., « Les choix des conjoints en agriculture », op. cit. 


\section{L'homogamie parentale et les deux voies d'entrée dans l'agriculture}

Nous allons voir comment la mixité sociale parentale influence les trajectoires scolaires et le choix de l'agriculture des fils et des filles d'agriculteur.

\section{L'bétérogamie parentale et la voie scolaire}

Le rapport à l'école des agriculteurs s'est considérablement transformé sous l'effet conjoint des transformations du système désignées par le terme de "massification " (allongement de la scolarité obligatoire voté en 1959, entrée massive des enfants de classes populaires au collège, multiplication des cursus techniques au collège) et du conditionnement des aides à l'installation à un niveau de diplôme minimal instauré en 1974. Les agriculteurs auparavant rapidement exclus du système scolaire ${ }^{14}$ se sont engagés dans le jeu de la compétition scolaire et ont rapidement obtenu, dans l'enseignement primaire comme au collège, des résultats meilleurs que les enfants d'ouvrier. Ils ont également fait plus fréquemment le choix délibéré, plus souvent masculin, à niveau de réussite scolaire égal, des filières techniques, par rapport aux enfants d'autres origines sociales ${ }^{15}$. Les filles d'agriculteur ont conservé, quant à elles, des choix de formations moins techniques et se sont orientées pendant cette première période de massification scolaire vers l'enseignement général. Quel rôle joue l'hétérogamie sur le rapport à l'école? Les choix scolaires des filles et des garçons étant très différents, nous distinguons ici les deux populations ${ }^{16}$.

\section{Les fils}

Pour la génération des hommes nés entre 1954 et 1963 (tableau 1), qui a connu l'allongement des études (passage de la scolarité obligatoire de 14 à 16 ans) et la mise en place au collège de nombreuses filières techniques ${ }^{17}$, l'hétérogamie parentale sur petite exploitation a un effet négatif sur la scolarité des fils car ils sont plus enclins que tous les autres à sortir du système scolaire sans aucun diplôme. Rapidement éliminés dans le processus

14. Jégouzo G., Brangeon J.-L., Les paysans et l'école, Paris, Cujas, 1976.

15. Euvrard F., Rondeau M.-C., "Déroulement de la scolarité des enfants d'agriculteurs ", Revue française de pédagogie, $\mathrm{n}^{\circ} 73$, oct.-déc., 1985, p.7-14.

16. La faiblesse de certains effectifs nous oblige à ne pas considérer comme significatifs les chiffres de la modalité "Grande/Moyenne exploitation hétérogame ». Cette modalité ne sera pas lue dans ces tableaux mais nous nous appuyons sur des comparaisons plus larges pour analyser l'effet de cette situation.

17. Nos données ne nous permettent pas de distinguer dans les différentes filières techniques celles qui sont spécifiquement agricoles. On se contentera donc juste d'une distinction entre les formations générales et techniques (sans autre indication). 


\begin{tabular}{|l|c|c|c|c|c|}
\hline & $\begin{array}{c}\text { Diplôme } \\
\text { du supérieur } \\
\text { et bac seul }\end{array}$ & $\begin{array}{c}\text { Diplôme } \\
\text { technique } \\
\text { du supérieur } \\
\text { ou bac } \\
\text { technologique } \\
\text { ou pro }\end{array}$ & $\begin{array}{c}\text { CAP, BEP, } \\
\text { Brevet } \\
\text { d'enseigne- } \\
\text { ment }\end{array}$ & $\begin{array}{c}\text { BEPC } \\
\text { ou CEP seul }\end{array}$ & $\begin{array}{c}\text { Aucun } \\
\text { diplôme }\end{array}$ \\
\hline $\begin{array}{l}\text { Grandes } \\
\text { exploitations } \\
\text { Homogames }\end{array}$ & 19,4 & 16,7 & 37,5 & 12,5 & 13,9 \\
$\begin{array}{l}\text { Grandes } \\
\text { exploitations }\end{array}$ & $(13,3)$ & $(6,7)$ & $(53,3)$ & $(20,0)$ & $(6,7)$ \\
$\begin{array}{l}\text { Hétérogames } \\
\text { Petites } \\
\text { exploitations } \\
\text { Homogames } \\
\begin{array}{l}\text { Petites } \\
\text { exploitations } \\
\text { Hétérogames }\end{array}\end{array}$ & 7,6 & 7,6 & 34,2 & 15,2 & 35,4 \\
\hline Effectifs & 53 & 74 & 237 & 83 & 149 \\
\hline
\end{tabular}

Source: Enquête FQP 2003.

Champ: Hommes de 40 à 49 ans ayant un père ou une mère exploitant agricole. $\mathrm{N}=596$. Lecture: 19,4\% des hommes de 40 à 49 ans dont les deux parents étaient agriculteurs sur une grande ou moyenne exploitation agricole ont obtenu un diplôme équivalent ou supérieur au bac.

Test du Khi2: significatif à $0,8 \%$.

TABLEAU 1. - Niveau de diplôme des hommes de 40 à 49 ans selon l'homogamie parentale et la dimension économique de l'exploitation des parents.

de sélection scolaire, ils partent rejoindre le monde ouvrier. Les fils de parents hétérogames sur grande exploitation ont plus tendance que ceux sur petite exploitation à avoir acquis un diplôme général de niveau bac ou supérieur ou bien à avoir suivi des études techniques courtes comme le CAP/BEP (les effectifs étant très faibles nous avons aussi vérifié cette tendance sur une classe d'âge plus importante).

Deux situations différentes se dessinent en matière d'hétérogamie: pour les petites exploitations elle peut correspondre à un contexte de difficultés économiques où le travail féminin est une source de revenu d'appoint pour la famille et l'exploitation. Dans ce contexte des années 1960 où le travail conjugal est la norme dominante, les femmes des petites exploitations ont pu chercher de quoi « faire bouillir la marmite ${ }^{18}$ ". Cette situation reflète donc des perspectives économiques difficiles pour les éventuels repreneurs

18. Barthez A., "Femmes actives hors de l'exploitation familiale ", in ARF, La pluriactivité dans les familles agricoles, Paris, ARF éditions, 1984, p. 211-222. 
qui peuvent désinciter un fils à s'investir dans l'agriculture. Mais elle résulte également d'une stratégie familiale, dans la mesure où elle vise à aider l'exploitation à continuer à fonctionner, la famille à vivre correctement. Cette situation signale donc aussi une mobilisation du collectif familial. L'hétérogamie du haut de l'échelle sociale agricole est différente: sur des exploitations dont la dimension économique est importante l'activité professionnelle des mères peut correspondre à un choix positif des femmes de disposer d'une carrière personnelle, sur le modèle des femmes des milieux urbains salariés, milieux dont elles peuvent être issues. Dans ce contexte, le couple parental peut offrir à ses enfants deux modèles professionnels assumés, une socialisation à deux mondes différents.

L'effet de l'homogamie parentale en revanche est assez proche quel que soit le type d'exploitation des parents. Si l'hétérogamie favorise le choix de cursus long de l'enseignement général (pour les grandes exploitations) ou les cursus courts ou les sorties sans diplôme de l'enseignement général (pour les petites exploitations), l'homogamie parentale favorise plus que toute autre situation familiale le choix des filières techniques. Les fils des moyennes et grandes exploitations comme des petites exploitations dont les parents sont tous deux agriculteurs s'orientent plus facilement vers un diplôme technique long (bac pro, BTS) que les enfants dont un seul parent est agriculteur; les fils de parents hétérogames sur petite exploitation sortent plus souvent sans diplôme que les autres enfants. L'effet de la dimension économique de l'exploitation est ici aussi manifeste puisqu'il permet aux fils des moyennes et grandes exploitations d'aller plus loin dans la formation générale.

Au final, la mixité sociale du couple parental explique plutôt la composition du capital scolaire des enfants, (le choix des filières techniques par rapport aux filières générales), alors que le capital économique des familles explique plutôt le volume de capital scolaire acquis par les enfants ${ }^{19}$.

Les filles

L'effet de l'hétérogamie chez les filles est moins net (tableau 2). De manière assez similaire aux garçons, les filles issues d'une petite exploitation et dont l'un des deux parents n'est pas agriculteur ont plus de risque $(47,6 \%)$ que celles dont les deux parents sont agriculteurs de quitter le système scolaire sans diplôme ou avec un diplôme de l'enseignement général court (CEP, BEPC).

Au vu des effectifs, la composition de la famille et le milieu agricole n'ont pas d'influence significative sur l'accès aux diplômes techniques courts (BEP, CAP) des filles (ce que l'on retrouve si l'on considère cette fois l'ensemble de la population et non plus cette classe d'âge). Le fait d'avoir

19. Giraud C., Rémy J, op. cit., 2013. 


\begin{tabular}{|l|c|c|c|c|c|}
\hline & $\begin{array}{c}\text { Diplôme } \\
\text { du supérieur } \\
\text { et bac seul }\end{array}$ & $\begin{array}{c}\text { Diplôme } \\
\text { technique } \\
\text { du supérieur } \\
\text { ou bac } \\
\text { technologique } \\
\text { ou pro }\end{array}$ & $\begin{array}{c}\text { CAP, BEP, } \\
\text { Brevet } \\
\text { d'enseigne- } \\
\text { ment }\end{array}$ & $\begin{array}{c}\text { BEPC } \\
\text { ou CEP seul }\end{array}$ & $\begin{array}{c}\text { Aucun } \\
\text { diplôme }\end{array}$ \\
\hline $\begin{array}{l}\text { Grandes } \\
\text { exploitations } \\
\text { Homogames }\end{array}$ & 17,3 & 26,7 & 22,7 & 16,0 & 17,3 \\
$\begin{array}{l}\text { Grandes } \\
\text { exploitations }\end{array}$ & $(5,3)$ & $(21,1)$ & $(31,6)$ & $(15,8)$ & $(26,3)$ \\
$\begin{array}{l}\text { Hétérogames } \\
\text { Petites } \\
\text { exploitations } \\
\text { Homogames }\end{array}$ & 15,8 & 15,6 & 25,9 & 21,5 & 21,2 \\
$\begin{array}{l}\text { Petites } \\
\text { exploitations } \\
\text { Hétérogames }\end{array}$ & 19,8 & 12,8 & 19,8 & 30,2 & 17,4 \\
\hline Effectifs & $\mathbf{9 2}$ & $\mathbf{8 7}$ & $\mathbf{1 3 3}$ & 118 & 109 \\
\hline
\end{tabular}

Source: Enquête FQP 2003.

Champ: Femmes de 40 à 49 ans ayant un père ou une mère exploitant agricole. $\mathrm{N}=539$. Lecture: 17,3\% des hommes de 40 à 49 ans dont les deux parents étaient agriculteurs sur une grande ou moyenne exploitation agricole ont obtenu un diplôme équivalent ou supérieur au bac.

Test du Khi2: significatif.

TABLEAU 2. - Niveau de diplôme des femmes de 40 à 49 ans selon l'homogamie parentale et la dimension économique de l'exploitation des parents.

deux parents ayant une activité professionnelle différente favorise l'accès à des diplômes de l'enseignement technique long (ce qui est vérifié sur l'ensemble de la population).

Enfin chez les familles homogames, avoir vécu dans une petite exploitation favorise les sorties du système scolaire avec un diplôme de l'enseignement général court ou pas de diplôme pour les filles quand vivre sur une exploitation moyenne ou grande favorise le choix de formations techniques longues (Bac Pro, BTS, premiers cycles universitaires).

Le poids des capitaux économiques joue donc ici surtout sur la durée des études (qu’elles soient techniques ou générales). Les études techniques (courtes) sont suivies de façon moins fréquentes que par les fils et ne paraissent pas influencées par la composition du couple de parents. Les études techniques longues (souvent indispensable pour reprendre une exploitation agricole) sont plus souvent le fait des filles venant des exploitations les plus grandes et dotées d'une socialisation homogène. 
La composition du couple parental et la position dans le milieu agricole des filles orientent différemment leur cursus scolaire: le plus souvent construites comme des personnes qui vont quitter le milieu agricole, elles vont plutôt obtenir un capital scolaire général faible (lorsqu'elles sont issues de petites exploitations homogames) ou plutôt élevé (si elles viennent de grandes exploitations homogames ou lorsqu'un de leurs parents a une activité professionnelle non agricole). Poursuivre des études permet aux jeunes femmes de se projeter vers une position professionnelle en dehors du milieu agricole. Mais, dans certains cas, les études peuvent constituer pour certaines filles qui souhaitent s'ancrer dans l'agriculture une position d'attente quand la situation autour de la succession professionnelle des parents n'est pas réglée ${ }^{20}$.

\section{L'bétérogamie parentale et le choix de l'agriculture}

Les chances de choisir l'agriculture sont très différentes selon l'homogénéité socio-professionnelle parentale et la dimension économique de l'exploitation des parents (tableau 3). Comme on pouvait s'y attendre les fils issus des exploitations grandes et moyennes ayant deux parents agriculteurs sont les plus enclins à rester agriculteurs. À l'inverse, les fils provenant des petites exploitations et dont un des deux parents seulement est agriculteur ont trois fois moins de chance de choisir l'agriculture que les précédents. Les deux facteurs (petite exploitation et hétérogamie parentale) se combinent ici. De la même manière, pour les femmes, qui choisissent bien moins souvent l'agriculture que les garçons, l'hétérogamie éloigne de l'agriculture tandis que l'homogamie en rapproche.

\begin{tabular}{|l|c|c|}
\hline & Hommes & Femmes \\
\hline Grandes exploitations Homogames & 30,3 & 10,6 \\
Grandes exploitations Hétérogames & 14,5 & 3,1 \\
Petites exploitations Homogames & 20,9 & 8,7 \\
Petites exploitations Hétérogames & 10,4 & 3,8 \\
\hline Effectifs & $\mathrm{N}=\mathbf{2 3 4 8}$ & $\mathrm{N}=2357$ \\
\hline Total & $\mathbf{2 0 , 5}$ & $\mathbf{8 , 0}$ \\
\hline
\end{tabular}

Source: Enquête FQP 2003.

Champ: Individus ayant un père ou une mère exploitant agricole.

Lecture: 36,4\% des hommes dont les deux parents sont ou étaient agriculteurs sur moyenne ou grande exploitation sont eux-mêmes agriculteurs.

TABLEAU 3. - Taux d'agriculteurs chez les enfants d'agriculteurs en \%, selon l'homogamie parentale et la dimension économique de l'exploitation des parents.

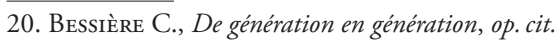


Plus surprenant, les fils et les filles venant de petites exploitations et dont les deux parents sont agriculteurs ont été plus enclins à choisir la profession d'agriculteur que les fils issus des exploitations grandes et moyennes dont un seul parent était agriculteur. Dans ces deux catégories les effets de la dimension économique de l'exploitation et de l'homogénéité professionnelle parentale se contrecarrent. L'effet de l'homogénéité du couple parental est même plus fort que celui de la dimension économique de l'exploitation: même sur une petite exploitation, qui offre aux enfants peu de perspectives économiques, l'homogamie parentale est parvenue à préserver un monde, une évidence, qui est moins souvent mise en doute que lorsque les deux parents ont des métiers différents. L'homogamie parentale a eu un effet positif sur la capacité de la petite agriculture du milieu des années 1950 et 1960, particulièrement mise à mal par les politiques agricoles et la crise de surproduction des années 1970 , à produire des enfants qui choisissent l'agriculture. Cet effet est notable également chez les filles. L'évidence d'une vie professionnelle dans le monde agricole se construit donc chez les filles sur les mêmes bases parentales. Deux parents agriculteurs orientent plus facilement vers l'agriculture qu'un seul parent. Nous allons voir maintenant que les filles accèdent également à la profession par un autre circuit que les fils.

\section{Du côté des filles: l'hétérogamie et la voie conjugale}

Le choix de l'agriculture se fait dans un contexte très différent pour les garçons ou pour les filles. Nombre de travaux ont pu montrer combien les filles, à la différence des garçons, étaient souvent mises à l'écart de la transmission des savoir-faire professionnels et, quand cette transmission s'est opérée, combien, dans le cadre de l'enseignement agricole ou des démarches administratives, les jeunes femmes étaient systématiquement dévalorisées, suspectées et devaient constamment faire leurs preuves ${ }^{21}$. Les jeunes femmes sont aussi plus souvent écartées par rapport à un frère dans le choix du successeur c'est-à-dire écartées du contrôle et de l'utilisation des capitaux professionnels familiaux. Les fils, déjà adoubés par la famille, doivent gérer au mieux les rapports à la profession et l'acquisition des titres scolaires nécessaires à une installation. Pour les filles, le choix de l'agriculture emprunte le plus souvent une autre voie quand elles ne sont pas choisies par leur famille: elles entrent dans la profession par le « mariage ». L'union avec un agriculteur leur permet d'accéder au métier d'agricultrice. Cette voie d'accès par le mariage est un chemin ancien; celui des femmes aides familiales qui ont participé toute leur vie et gratuitement

21. Rieu A. et Dahache S., « S'installer comme agricultrice: sur la socialisation et la formation sexuée en agriculture ", Revue d'études en agriculture et environnement, n³ 3, 2008, p. 71-94; Bessière C., De génération en génération, op.cit. 
au travail de l'exploitation familiale. Certes, les filles d'agriculteur ont de plus en plus souhaité acquérir une activité professionnelle et quitter le monde de l'agriculture au risque de condamner les jeunes agriculteurs au célibat ${ }^{22}$.

Pourtant, cette voie ancienne ne s'est pas tarie. Les rapports sociaux de couple et les statuts professionnels en agriculture se sont profondément transformés depuis les années 1970: les femmes ne sont plus obligées comme avant d'épouser le métier de leur mari. Pour certaines femmes à qui la succession familiale est refusée, le choix d'un agriculteur comme mari, puis de l'agriculture comme métier peut être vécu, dans un contexte d'affaiblissement du modèle professionnel de travail agricole conjugal, plus comme une opportunité que comme une contrainte. Les statuts professionnels des femmes en agriculture permettent aujourd'hui à celles qui travaillent avec leur conjoint d'obtenir les mêmes avantages, les mêmes droits sociaux, le même statut professionnel. Ce choix des filles d'agriculteur d'une profession, via le mariage, est donc très différent de celui de leurs mères, contraintes d'aider leur mari sans espoir de reconnaissance professionnelle ou sociale de leur travail.

\begin{tabular}{|l|c|c|}
\hline & Taux & Effectifs totaux \\
\hline Grandes exploitations Homogames & 14,3 & 358 \\
Grandes exploitations Hétérogames & 9,2 & 98 \\
Petites exploitations Homogames & 12,9 & 1536 \\
Petites exploitations Hétérogames & 6,6 & 365 \\
\hline Ensemble & 12,0 & 2357 \\
\hline
\end{tabular}

Source: FQP 2003.

Champ: Filles d'agriculteur.

Test du Khi2: significatif au seuil de 0,1\%.

TABLEAU 4. - Hétérogamie et choix d'un conjoint agriculteur par les filles d'agriculteur 23.

Si l'homogamie parentale protège l'évidence sociale qui fait de l'agriculture le monde social et professionnel par excellence pour les enfants, l'homogamie doit favoriser aussi le choix pour les filles d'un conjoint agriculteur et de la profession agricole aux côtés du mari. Le tableau suivant, le confirme: les filles qui ont deux parents agriculteurs ont plus tendance à vivre en couple avec un agriculteur que celles qui ont un seul parent agriculteur.

Mais l'influence du milieu social parental ne s'arrête pas au choix conjugal, car le choix d'un conjoint agriculteur permet à des femmes

22. Bourdieu P., Le bal des célibataires, Paris, Seuil, 2002.

23. Pour des raisons d'effectifs, nous avons choisi de ne pas nous limiter aux seules femmes quarantenaires mais nous avons considéré l'ensemble de la population des filles d'agriculteur. 
dépourvues de capitaux fonciers propres de devenir agricultrices (avec des statuts variables): 46,5\% des femmes en couple avec un agriculteur se déclarent agricultrices contre 1,8\% des femmes en couple avec un homme qui n'est pas agriculteur ${ }^{24}$.

\begin{tabular}{|l|c|c|c|c|}
\hline & $\begin{array}{c}\text { Choix } \\
\text { de l'agriculture } \\
\text { par les femmes } \\
\text { en couple avec } \\
\text { un agriculteur }\end{array}$ & $\begin{array}{c}\text { Effectifs } \\
\text { totaux }\end{array}$ & $\begin{array}{c}\text { Choix } \\
\text { de l'agriculture } \\
\text { par les femmes } \\
\text { en couple avec } \\
\text { une personne } \\
\text { qui n'est pas } \\
\text { agriculteur }\end{array}$ & $\begin{array}{c}\text { Effectifs } \\
\text { totau }\end{array}$ \\
\hline $\begin{array}{l}\text { Grandes } \\
\text { exploitations }\end{array}$ & 52,9 & 51 & 3,1 & 223 \\
$\begin{array}{l}\text { Homogames } \\
\text { Grandes } \\
\text { exploitations } \\
\text { Hétérogames }\end{array}$ & 22,2 & 9 & 1,7 & 59 \\
$\begin{array}{l}\text { Petites } \\
\text { exploitations } \\
\text { Homogames }\end{array}$ & 47,5 & 198 & 1,7 & 1020 \\
$\begin{array}{l}\text { Petites } \\
\text { exploitations } \\
\text { Hétérogames }\end{array}$ & 33,3 & 24 & 1,2 & 243 \\
\hline Ensemble & 46,5 & 282 & 28 & 1545 \\
\hline Effectif & 131 & & & \\
\hline
\end{tabular}

Source: FQP 2003

Champ: Filles d'agriculteurs

Test du Khi2: Pas significatif en raison des faibles effectifs.

TABLEAU 5. Hétérogamie et choix de la profession par les femmes (selon la profession du mari), en \%.

L'influence de l'homogamie parentale est à nouveau manifeste dans le choix du métier d'agricultrice: pour les femmes qui ont épousé un agriculteur comme pour celles qui vivent avec hommes qui ne l'est pas, le fait d'avoir deux parents agriculteurs favorise davantage le choix professionnel de l'agriculture que le fait de n'avoir qu'un parent agriculteur. Le fait d'être issu d'une grande exploitation et d'avoir deux parents agriculteurs favorise

24. On sait combien les successions " en gendre » sont aujourd'hui une figure du passé (Bessière C., op. cit., 2010). Les femmes qui succèdent à leurs parents ont des conjoints masculins qui conservent plus facilement une profession indépendante de l'exploitation agricole. La montée des agricultrices est ambiguë: les agricultrices, chefs d'exploitation sans conjoint, sont finalement peu nombreuses (sur l'ensemble de la population), la part la plus nombreuse reste encore celle des femmes pour qui le mariage ou le couple a ouvert la porte d'une activité professionnelle sur une exploitation agricole. 
le plus le choix de la profession d'agricultrice que ce soit en couple ou en solo ${ }^{25}$.

Pour les générations les plus récentes en effet, la capacité pour les épouses à travailler à l'extérieur, fait du choix de devenir agricultrice aux côtés d'un mari une situation moins contrainte qu'avant. Il s'agit plus d'une décision qui peut se faire sous condition et qui peut être éventuellement réversible (dans la mesure où les jeunes femmes sont pourvues de capitaux scolaires). De plus l'amélioration du statut professionnel des conjointes en agriculture les protège davantage que les conjointes des générations précédentes. La profession d'agricultrice se présente aujourd'hui davantage comme un choix personnel qui peut être vécu comme épanouissant que comme un triste destin associé au mariage.

\section{Synthèse}

Pour conclure cette première partie, nous avons essayé de montrer les effets croisés et cumulatifs de la socialisation familiale, de la socialisation scolaire et des choix conjugaux. Pour ce faire, nous avons réalisé une régression logistique sur l'ensemble des fils d'agriculteur et une seconde sur les filles afin de voir les effets qui pèsent le plus sur le choix de l'agriculture comme profession.

Les fils

Toutes choses du modèle égales par ailleurs, le fait d'avoir deux parents agriculteurs agit plus favorablement, que l'on soit issu d'une petite ou d'une moyenne ou grande exploitation sur le choix de l'agriculture par l'enfant que le fait d'avoir un seul parent agriculteur sur petite exploitation (tableau 6). En matière de maintien dans l'agriculture, l'homogamie parentale protège du facteur défavorable que représente le fait d'être issu d'une petite exploitation.

Deuxième point notable, les chances de rester agriculteur, comme la génération précédente, sont plus fortes pour les enfants disposant d'une formation technique (comme le BEP ou mieux encore le Bac Pro). Le fait de n'avoir aucun diplôme est relativement moins propice à un choix

25. Notons que l'homogamie parentale favorise également les unions des fils avec une conjointe agricultrice. Là encore le modèle parental constitue un élément très fort pour les enfants des deux sexes. Cette situation n'est pas dénuée d'ambiguïté: volonté des fils de reproduire les modèles familiaux? Pression des parents pour trouver une épouse qui puisse travailler aux côtés du fils qui s'installe? Certains jeunes agriculteurs qui anticipent les risques de célibat une fois installés en agriculture vont parfois mettre à profit leurs expériences de détour professionnel après leurs études pour faire couple et dans un deuxième temps s'installer. Il est difficile d'évaluer l'ampleur de ces stratégies, et encore moins leur réussite puisque certains fils d'agriculteurs peuvent reporter durablement leur projet d'installation en agriculture en raison de négociations conjugales difficiles. Les liens entre choix du conjoint et installation en agriculture sont donc complexes. 


\begin{tabular}{|l|c|c|c|}
\hline & Odds ratio & Coefficient & Significativité \\
\hline Grandes exploitations Homogames & 3,191 & 1,1605 & $* * *$ \\
Grandes exploitations Hétérogames & - & - & $\mathrm{ns}$ \\
Petites exploitations Homogames & 2,235 & 0,8043 & $* * *$ \\
Petites exploitations Hétérogames & ref & ref & \\
\hline Aucun diplôme & ref & ref & \\
CEP ou BEPC seul & - & - & $\mathrm{ns}$ \\
CAP, BEP & 2,469 & 0,9038 & $* * *$ \\
Diplôme technique sup, bac technique & 3,536 & 1,2631 & $* *$ \\
Diplôme universitaire ou Bac général & - & - & $\mathrm{ns}$ \\
\hline Conjointe agricultrice & 15,333 & 2,73 & $* * *$ \\
Conjointe pas agricultrice & ref & ref & $*$ \\
Pas d'information sur la conjointe & 2,171 & 0,7752 & $* * *$ \\
\hline
\end{tabular}

Modèle explicatif ${ }^{6}$ : modèle Logit expliquant la probabilité de devenir agriculteur. Source: Enquête FQP 2003.

Champ: Hommes ayant son père ou sa mère exploitant agricole. $N=2348$ individus.

TABLEAU 6. - Probabilité de se maintenir dans l'agriculture pour les fils (régression logistique).

professionnel agricole par l'enfant. Même moins fréquente, c'est cependant une trajectoire professionnelle bien réelle. Il est possible ainsi de distinguer deux profils d'enfants qui restent dans l'agriculture: ceux qui sont dotés d'un fort capital scolaire technique et qui vont entrer en agriculture en bénéficiant des aides à l'installation offertes par la profession; ceux qui ne disposent que d'un capital scolaire très réduit et se trouvent dans une situation moins favorable pour s'installer. Ces derniers peuvent trouver dans l'exploitation familiale un recours pour éviter le salariat dans le monde ouvrier.

L'évidence de la succession ne se construit pas de la même manière dans les deux cas: dans le cas des familles de la petite agriculture, l'évidence s'appuie sur un vécu qui est lié à la famille, au travail quotidien. Cette construction est de moins en moins évidente dans la mesure où elle s'accompagne d'un rejet de la part de l'appareil d'encadrement agricole qui va rendre l'installation moins simple, moins légitime. Dans le cas des familles

26. La probabilité qu'un coefficient soit non nul est, selon les cas, inférieure à $0,1 \%$ (notée ${ }^{* * *}$ ) ou comprise entre $0,1 \%$ et $1 \%$ (noté ${ }^{* *}$ ) ou comprise entre $1 \%$ et $5 \%$ (noté ${ }^{*}$ ) ou supérieure (notée ns). Un coefficient positif indique une augmentation de la probabilité de la modalité correspondante par rapport à la probabilité de la modalité de référence alors qu'un coefficient négatif indique une baisse de cette même probabilité. Un odds-ratio supérieur à 1 indique un rapport de chance favorable par rapport à la modalité de référence. Inférieur à 1, l'odds ratio indique une moindre chance par rapport à la modalité de référence de devenir agriculteur. 
plus aisées, l'évidence s'appuie sur des stratégies scolaires longues, où l'enfant qui choisit l'agriculture est " adoubé " par la profession. L'évidence se construit dans l'interaction avec plusieurs institutions (famille, école, profession agricole) ${ }^{27}$.

Enfin avoir une conjointe agricultrice est fortement lié au fait d'être agriculteur mais le lien est difficile à interpréter pour les hommes. De même le fait de ne pas avoir d'information sur la profession de la conjointe (ce qui signifie le plus souvent qu'elle n'existe pas, que le fils est célibataire) est aussi à mettre en lien avec le choix de l'agriculture. Ici encore les effets sont difficiles à interpréter mais il semble clair que le célibat est plus un effet de l'installation en tant qu'agriculteur qu'une cause.

\section{Les filles}

Toutes choses du modèle égales par ailleurs, pour les filles, avoir deux parents agriculteurs sur grande ou moyenne exploitation favorise le choix de l'agriculture, par rapport au fait d'avoir un seul parent agriculteur sur petite exploitation (tableau 5). Pour les filles, l'homogamie parentale favorise le choix de l'agriculture surtout lorsque la socialisation s'est faite sur une exploitation de taille économique importante.

Le fait de posséder un diplôme technique (Bac Pro, BTS) ou de premier cycle universitaire semble défavoriser le choix de l'agriculture comme profession par rapport au fait de ne pas avoir de diplôme.

\begin{tabular}{|l|c|c|c|}
\hline & Odds ratio & Coefficient & Significativité \\
\hline Grandes exploitations Homogames & 2,145 & 0,7631 & $*$ \\
Grandes exploitations Hétérogames & - & - & $\mathrm{Ns}$ \\
Petites exploitations Homógames & - & - & $\mathrm{Ns}$ \\
Petites exploitations Hétérogames & ref & ref & \\
\hline Aucun diplôme & ref & ref & \\
CEP ou BEPC seul & - & - & $\mathrm{Ns}$ \\
CAP, BEP & - & - & $\mathrm{Ns}$ \\
Diplôme technique sup, bac technique & 0,237 & $-1,4385$ & $* * *$ \\
Diplôme universitaire ou Bac général & - & - & $\mathrm{ns}$ \\
\hline Conjoint agriculteur & 47,820 & 3,8674 & $* * *$ \\
Conjoint pas agriculteur & ref & ref & \multirow{2}{*}{ Pa** } \\
Pas d'information sur la conjoint & 3,403 & 1,2246 & \\
\hline
\end{tabular}

Modèle explicatif: modèle Logit expliquant la probabilité de devenir agricultrice.

Source: Enquête FQP 2003.

Champ: Hommes ayant son père ou sa mère exploitant agricole. $\mathrm{N}=2288$ individus

TABLEAU 7 - Probabilité de se maintenir dans l'agriculture pour les filles (régression logistique).

27. Sur tous ces points, voir Giraud C., Rémy J., op. cit, 2014. 
L'effet le plus important dans cette régression sur la population des femmes est celui du choix conjugal. Être en couple avec un agriculteur favorise le choix de l'agriculture comme métier par rapport au fait d'être en couple avec une personne ayant une autre profession. Nous avons vu pourtant que certaines femmes choisissaient le métier d'agricultrice tout en étant en couple avec une personne ayant un autre métier. Mais elles sont trop peu nombreuses au sein de notre population pour être visibles dans cette régression par rapport au lien très fort existant entre le choix d'un conjoint agriculteur et le choix du métier d'agricultrice ${ }^{28}$.

Ces deux régressions montrent combien l'entrée dans le métier d'agriculteur se fait de façon différente pour les filles et les fils d'agriculteurs. L'effet "pur " de l'homogamie parentale est plus ou moins visible dans ces régressions (évident chez les garçons, moins net chez les filles), mais nous ne devons pas oublier que l'hétérogamie influence aussi le choix des études et le choix du conjoint. À travers le choix féminin d'un petit ami agriculteur, la trace d'une socialisation familiale cohérente est très nette.

\section{Distance sociale conjugale, cursus scolaire et choix de l'agriculture}

L'hétérogamie parentale a des effets importants sur le devenir scolaire, sur les choix professionnels ou les choix matrimoniaux, mais elle recouvre des réalités très différentes : avoir une mère ouvrière ou employée n'a évidemment pas le même effet qu'avoir une mère cadre. L'appartenance professionnelle de l'épouse ${ }^{29}$ et la distance sociale par rapport au monde agricole pèse différemment sur la socialisation des enfants et sur leurs choix. Nous souhaitons ici mesurer l'impact de la position sociale relative des deux parents sur le cursus scolaire et le choix de l'agriculture par leurs enfants.

\section{L'appartenance professionnelle de la mère et le cursus scolaire des enfants}

Le cursus des enfants change fortement lorsque la mère dispose d'une profession qui est éloignée socialement (cadres, professions intermé-

28. Rappelons que les régressions portent sur l'ensemble de la population. Des études récentes semblent montrer que les jeunes femmes de milieu agricole ou non-agricole s'investissent très fortement au sein de l'enseignement agricole et forment des projets d'installation agricole sans aucune considération pour leur situation conjugale future. Conscientes des faibles chances de trouver un conjoint masculin collaborateur sur leur projet, elles affichent une volonté de séparer leur activité agricole de l'activité professionnelle de leur mari, à l'image des couple biactifs (DAHACHE S., La féminisation de l'enseignement agricole, Paris, L'Harmattan, 2012, p. 90-119).

29. Dominique Jacques-Jouvenot insiste sur le fait que les mères qui ont une activité professionnelle non agricole sont celles qui encouragent le plus les enfants à effectuer des études mais aussi à ne pas choisir l'agriculture pour orientation professionnelle (JACQUes-Jouvenot D., Choix du successeur et transmission patrimoniale, Paris, L'Harmattan, 1997, p. 118). 
diaires, indépendants non agricole) de l'agriculture et qui peut se poser comme une alternative valorisante à l'agriculture. Les mères qui ont les positions sociales les plus aisées renforcent les choix scolaires de filières générales et longues (qui vont favoriser chez les fils une sortie de l'agriculture "par le haut»). Par contre, les mères appartenant au monde des employées (sans doute proche des activités de service aux personnes) ou même les mères sans profession, favorisent les sorties sans diplôme et le choix de filières techniques courtes (comme le CAP) qui impliquent plus souvent des sorties vers le monde ouvrier.

Les fils d'ouvrières, d'agricultrices ou de mères sans profession se sont tournés plus que les autres vers les filières techniques (comme le BEP ou le bac pro), sésame indispensable, pour cette génération, pour l'accès aux aides à l'installation (graphique 1). Les profils scolaires des fils d'ouvrière ou des fils d'agricultrice sont donc finalement très proches. L'agriculture reste pour ces deux profils de fils un choix que leur scolarité rend possible.

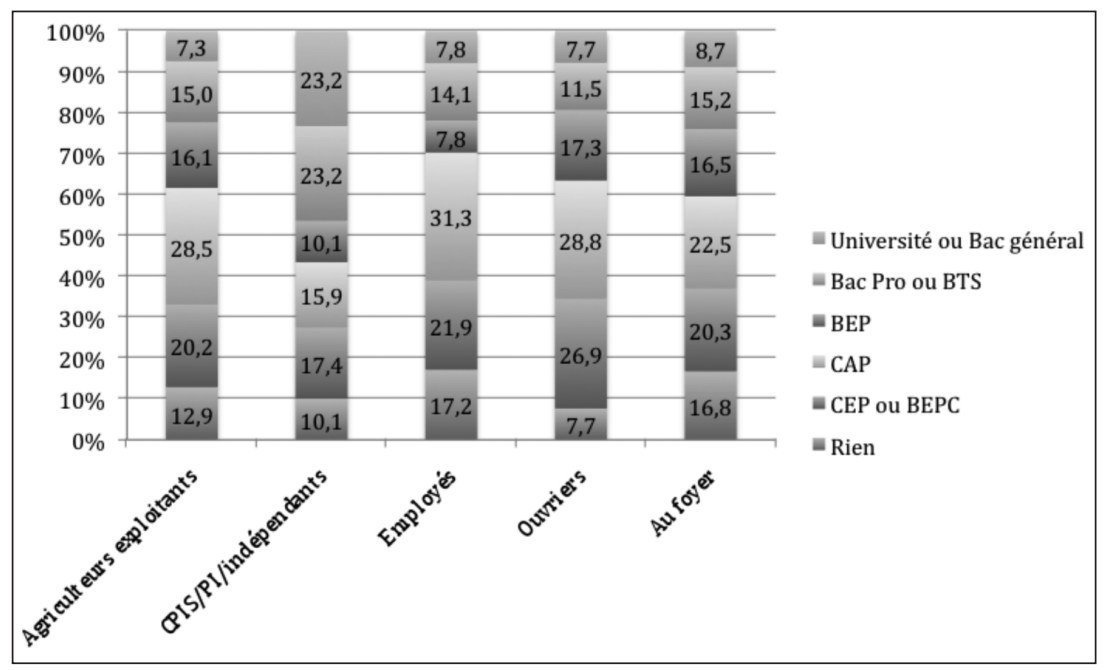

Source: Enquête EHF 1999.

Champ: Hommes, fils d'agriculteurs âgés de 40 à 49 ans. N $=4005$.

Lecture: $12,9 \%$ des fils d'agriculteurs âgés de 40 à 49 ans ayant une mère exploitante ont quitté l'école sans diplôme.

GRAPHIQUE 1. - Position sociale de la mère et capital scolaire des fils.

Pour ce qui est des filles (graphique 2), le fait d'avoir une mère ouvrière est la situation qui a le plus favorisé les sorties du système scolaire avec un niveau de diplôme faible (BEPC ou CAP). Avoir une mère agricultrice ou au foyer favorise le choix de cursus techniques et généraux plus longs, davantage que le fait d'être fils d'ouvrière. Enfin, avoir une mère employée ou de milieu supérieur (notamment indépendante) favorise le plus les 


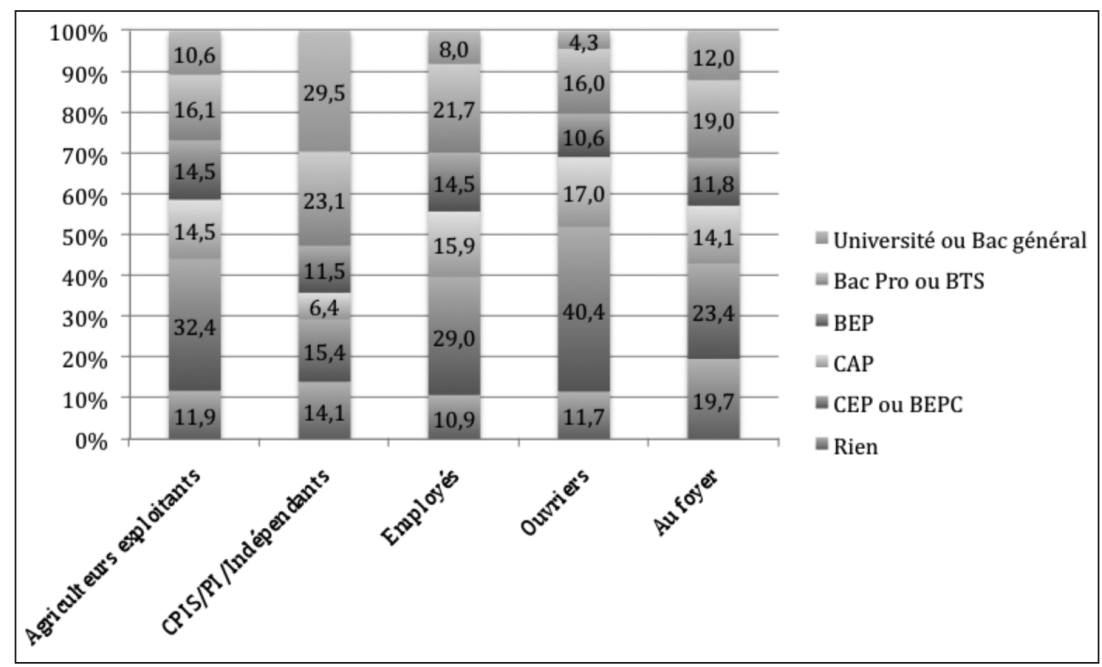

Source: Enquête EHF 1999.

Champ: Hommes, fils d'agriculteurs âgés de 40 à 49 ans. N = 5893.

Lecture: $11,9 \%$ des fils d'agriculteurs âgés de 40 à 49 ans ayant une mère exploitante ont quitté l'école sans diplôme.

GRAPHIQUE 2. - Position sociale de la mère et capital scolaire des filles.

cursus techniques longs (bac pro, BTS) ou généraux longs (bac ou fac). Finalement plus la mère est située haut dans l'échelle sociale, meilleur est le cursus de leurs filles. Les filles de mère agricultrices ou au foyer ont, quant à elles, des cursus qui sont moyens par rapport aux autres.

D'une certaine manière, les filles se différencient ici des garçons par le rejet relatif des filières techniques (comme le BEP ou le CAP), ce qui est une traduction du fait qu'elles ne se destinent pas à choisir l'agriculture comme profession. Elles s'en distinguent aussi par l'importance des filières longues (générales avec le niveau bac ou fac ou techniques avec le bac pro ou le BTS $)^{30}$. Mais les différences sont notables aussi en ce qui concerne l'ascendance ouvrière des filles et des garçons. La mère ouvrière " plombe " la scolarité des filles en la limitant plus souvent à des niveaux inférieurs à ceux obtenus par les filles d'agricultrices alors que, pour les garçons, elle permet de faire les mêmes études que les fils d'agricultrices.

\section{Le choix de l'agriculture et la profession de la conjointe}

La position sociale de l'épouse et la distance qui la sépare de son conjoint ont aussi des répercussions sur le choix de l'agriculture comme profession par

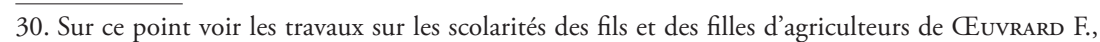
Rondeau M.-C., "Déroulement de la scolarité des enfants d'agriculteurs », op. cit. et Davaillon A., "Parcours scolaires des élèves ruraux et des enfants d'agriculteurs: spécificités et évolutions ", Éducation et formations, ${ }^{\circ}$ 54, déc., 1998, p. 97-107. 
les enfants. Les résultats obtenus sont cependant surprenants : quelle que soit la taille de l'exploitation du père, avoir une mère agricultrice a favorisé plus fortement que tout autre profil le choix de l'agriculture. L'exemple maternel, assure une forme d'évidence qu'aucune autre identité sociale ne peut égaler.

Pour les petites exploitations, le fait d'avoir une mère ouvrière ou indépendante favorise le choix de l'agriculture davantage que celui d'avoir une mère cadre, employée ou sans profession. La distance sociale par rapport à la petite agriculture (mère cadre ou profession intermédiaire...) favorise davantage le départ de l'agriculture qu'une ascendance maternelle proche socialement (indépendance, appartenance au monde ouvrier et surtout appartenance à l'agriculture). L'absence de profession déclarée de la mère conduit plus souvent qu'une ascendance agricole ou ouvrière à une sortie de l'agriculture. Pour les exploitations moyennes et grandes, le fait d'avoir une mère cadre, ouvrière, ou sans profession favorise davantage le choix de l'agriculture que le fait d'avoir une mère employée ou indépendante. Plus élevée que dans les petites exploitations, la profession du père est ici relativement plus attractive, même par rapport à des emplois féminins de cadre ou professions intermédiaires, et évidemment d'ouvrière.

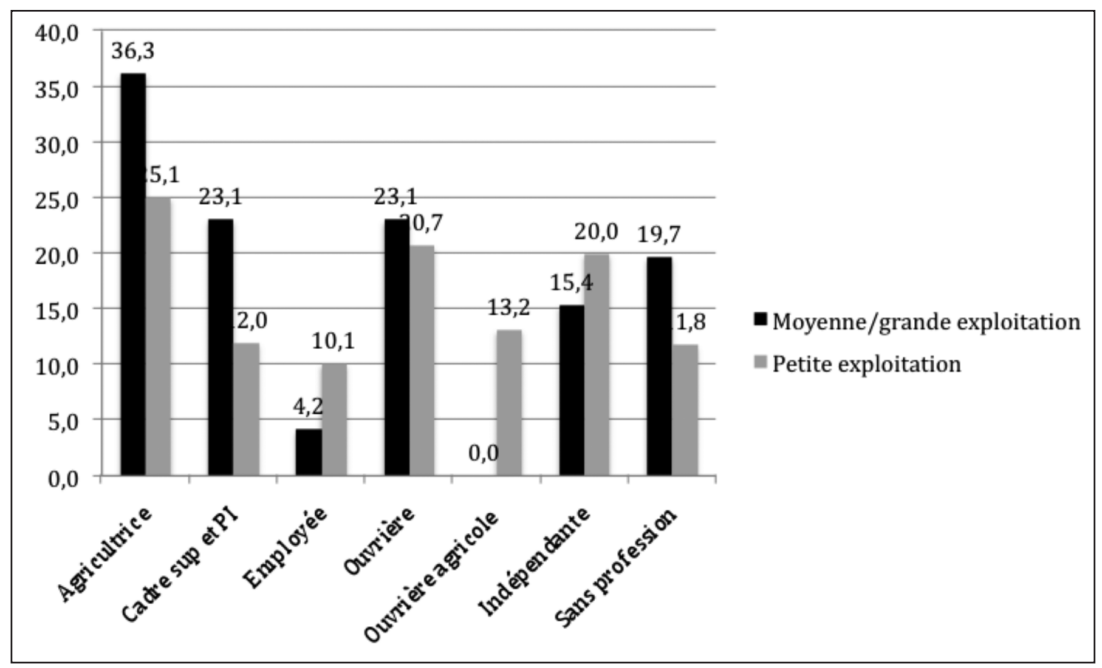

Source: Enquête FQP 2003.

Champ: Hommes ayant un père exploitant agricole ${ }^{31}$. Sur moyenne et grande exploitation. $\mathrm{N}=370$. Sur petite exploitation: $\mathrm{N}=1791$.

Lecture: $36,3 \%$ des fils d'agriculteur sur moyenne et grande exploitation dont la mère était agricultrice ont choisi le métier d'agriculteur.

GRAPHIQUe 3. - Choix de l'agriculture par les fils d'agriculteurs selon la taille de l'exploitation du père et selon la profession de la mère.

31. Pour des raisons d'effectifs, nous avons choisi de ne plus nous limiter à la classe d'âge des quarantenaires. 
Dans le cas des petites exploitations, la position sociale faible du père met celui-ci dans une posture sociale défavorable par rapport à une mère salariée. Les fils (graphique 3) choisissent alors moins souvent le métier d'agriculteur lorsque la position de la mère est distante (et plus élevée) que celle du père. Dans les grandes exploitations en revanche, la position sociale paternelle est plus élevée et le choix de l'agriculture par les fils peut être relativement fréquent même lorsque la mère dispose d'une profession élevée (comme c'est le cas pour les mères cadres ou professions intermédiaires). Dans les deux milieux agricoles, la position de fils d'ouvrière favorise davantage les sorties de l'agriculture que la position de fils d'agricultrice mais moins que la position de fils d'employée. Si les choix scolaires des fils d'ouvrières sont proches de ceux des fils d'agricultrices, au moment de choisir la profession, l'origine sociale mixte des premiers les conduit plus facilement à s'éloigner de l'agriculture.

Pour les femmes (graphique 4), filles d'un agriculteur travaillant sur petite exploitation, l'influence de la profession maternelle est aussi claire: avoir une mère agricultrice est la seule configuration familiale qui favorise

le choix par les filles de l'agriculture, quelle que soit la taille de l'exploitation. En second lieu, les mères indépendantes favorisent davantage le choix de l'agriculture que les autres profils. Bref, avoir une mère qui occupe

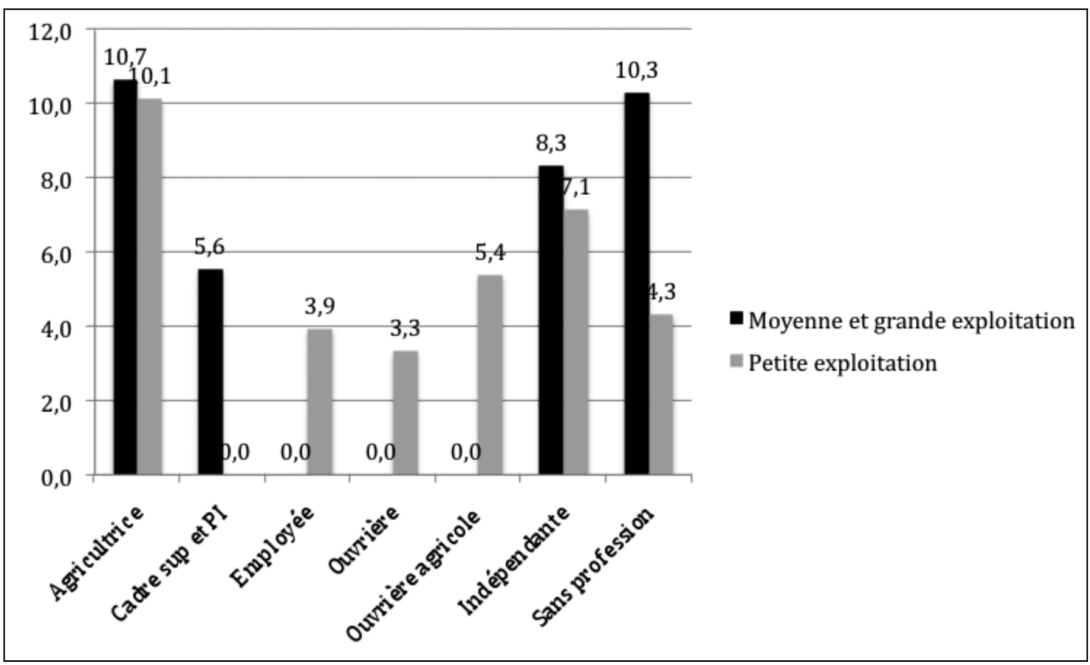

Source: Enquête FQP 2003.

Champ: Femmes ayant un père exploitant agricole. $\mathrm{N}=403$ pour les moyennes et grandes exploitations, $\mathrm{N}=1736$ pour les petites exploitations.

Lecture: $10,7 \%$ des filles d'agriculteur sur grande exploitation dont la mère était agricultrice ont choisi le métier d'agricultrice.

GRAPHIQUE 4. - Choix de l'agriculture par les filles d'agriculteurs selon la taille de l'exploitation du père et selon la profession de la mère. 
une activité salariée, modeste ou importante, détourne les filles de l'agriculture quand la position sociale du père est, elle, modeste.

Dans les moyennes ou grandes exploitations, avoir une mère agricultrice, sans profession ou indépendante sont des configurations qui favorisent le choix de l'agriculture par les filles. La distance sociale entre les positions paternelle et maternelle est réduite. Tout emploi salarié de la mère en revanche conduit les filles à choisir plutôt de quitter l'agriculture. Il semble qu'ici le modèle paternel est d'autant plus suivi que l'identité sociale des mères ne s'en éloigne pas trop.

Plus la distance sociale entre conjoint est réduite et plus les enfants (filles ou garçons) choisissent l'agriculture comme profession. Lorsqu'une distance sociale existe, les positions relatives des conjoints influencent les choix des enfants. Pour les filles, l'exemple maternel de travail salarié à l'extérieur les conduit à s'éloigner de la profession agricole, que les exploitations soient grandes ou petites ${ }^{32}$. Les grandes exploitations protègent les fils d'exemples maternels valorisants de travail à l'extérieur qui pourraient les détourner de l'agriculture, alors que les petites exploitations ne donnent une image valorisante de l'agriculture que lorsque la mère est ouvrière ou indépendante.

\section{Conclusion}

Le développement croissant de l'hétérogamie dans les couples en agriculture pose de façon centrale la question de la reproduction en agriculture. Nous avons pu montrer combien les enfants qui ont deux parents agriculteurs ont plus de chance que ceux qui n'en ont qu'un seul de choisir la profession d'agriculteur ou d'agricultrice. Ce lien, valable, quelle que soit la taille de l'exploitation et quel que soit le sexe de l'enfant, permet de comprendre pourquoi certaines petites exploitations ont pu perdurer et se reproduire en dépit de conditions économiques qui se sont durcies durant la période étudiée et ont conduit à une élimination importante des plus petites exploitations ${ }^{33}$.

La distance sociale qui peut exister entre les parents doit être mise en relation avec la dimension économique de l'exploitation des parents et le sexe des enfants: ainsi sur petite exploitation, l'activité professionnelle salariée de la mère a constitué pour les filles un exemple attractif et a contribué à les encourager à prendre elles aussi un travail salarié. C'est la même chose pour les garçons, à ceci près que la position d'ouvrière de leur

32. Comme nous l'avons montré en première partie, ce fait est étroitement lié aux choix conjugaux. Les filles dont la mère avait une activité professionnelle salariée ont plus tendance que les autres à être en couple avec un homme qui n'est pas agriculteur. Les filles dont la mère est agricultrice sont celles qui ont le plus tendance à s'unir avec un homme agriculteur.

33. Giroux G., "Recensement agricole 2010, Premières tendances ", Agreste - Recensements agricoles, $\mathrm{n}^{\circ} 266,2011$. 
mère semble moins attractive socialement pour eux que la position agricole de leur père. Sur moyenne ou grande exploitation, les choses sont différentes car la distance doit se mesurer à partir d'une position du père qui est plus élevée. Au regard de l'importance économique de l'exploitation agricole, l'activité professionnelle salariée des mères n'est pas forcément attractive pour les hommes. La croyance dans la profession agricole se raffermit, ce d'autant plus que les voies scolaires permettent davantage de bénéficier d'aides à l'installation. Pour les filles, en revanche, l'exemple maternel d'une activité salariée fonctionne comme un modèle positif qui les pousse, comme dans les petites exploitations, à ne pas choisir l'agriculture. Seuls les modèles maternels de l'agricultrice ou de la mère dite « sans profession » ou " indépendante » incitent les filles des grandes exploitations à choisir conjugalement, puis professionnellement, l'agriculture.

Cette analyse rapide nous amène à poser plus largement la question des capitaux dont disposent les familles en agriculture et la façon dont elles les transmettent et les font fructifier sur les marchés scolaires, conjugaux et professionnels. L'augmentation de l'hétérogamie, et avec elle, l'augmentation des capitaux sociaux et scolaires peut laisser supposer que les agriculteurs peuvent disposer désormais tant de capitaux économiques que de capitaux scolaires et qu'ils sont à même aujourd'hui de choisir pour leurs enfants de se reproduire hors de l'agriculture dans des professions salariées valorisantes. Cela pose aussi la question des stratégies que les agriculteurs développent pour construire des individus qui vont choisir, de façon positive, de vivre et travailler sur une exploitation agricole. Si l'agriculture était au $\mathrm{XIX}^{\mathrm{e}}$ siècle une condition sociale, elle est devenue une profession qui est destinée à être choisie par ses membres. Cette nouvelle liberté suppose donc toute une série de mécanismes familiaux et sociaux visant à orienter et à limiter ce choix. 